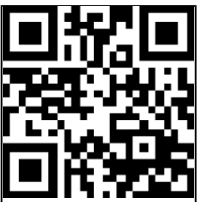

Editor's choice

Scan to access more free content

${ }^{1}$ Department of Philosophy, University of Massuchessetts, Boston, USA

${ }^{2}$ Department of Social Science, Health \& Medicine, King's College London, London, UK

Correspondence to Dr Annette Rid, Department of Social Science, Health \& Medicine, School of Social Science \& Public Policy, King's College London, Strand, London WC2R 2LS, UK ; annette.rid@kcl.ac.uk

Received 3 November 2013 Revised 2 May 2014 Accepted 23 September 2014 Published Online First 28 October 2014

\title{
Can informed consent to research be adapted to risk?
}

\author{
Danielle Bromwich, ${ }^{1}$ Annette Rid $^{2}$
}

\begin{abstract}
The current ethical and regulatory framework for research is often charged with burdening investigators and impeding socially valuable research. To address these concerns, a growing number of research ethicists argue that informed consent should be adapted to the risks of research participation. This would require less rigorous consent standards in low-risk research than in high-risk research. However, the current discussion is restricted to cases of research in which the risks of research participation are outweighed by the potential clinical benefits for the individual research participant. Furthermore, current proposals do not address the concern that risk-adapted informed consent may result in enrolling participants into research without their autonomous authorisation. In this paper, we show how the standard view of informed consent-consent as autonomous authorisation - can be adapted to risk even when the research does not have a favourable riskbenefit profile for the participant. Our argument has two important implications: first, it implies that current and proposed consent standards are not adequately calibrated to risk and, second, that consent standards also need to be adapted to factors other than risk.
\end{abstract}

\section{INTRODUCTION}

Biomedical research exposes participants to risks primarily for the benefit of others. It is widely held that this is ethically permissible only if competent participants have given valid informed consent and other ethical requirements for research are met. ${ }^{1}$ On the standard view, consent is valid when a competent adult receives relevant information about the study and adequately understands this information before voluntarily agreeing to enrolment. ${ }^{2-4}$ This view is enshrined in most research regulations and guidelines. ${ }^{15-8}$

On the standard view, investigators are responsible for ensuring that the conditions for valid consent-competence, disclosure, understanding and voluntariness-are met. For example, the Council for International Organisations of Medical Sciences Guidelines state that 'by informing the potential subjects... and by ensuring that each individual understands each procedure, investigators elicit their informed consent....'5 But this view seems to place quite a burden on investigators. They need to ensure that information is disclosed in an understandable way and must also guarantee that enrolment decisions are based on a thorough understanding of that information and are free from coercion and undue influence. These standards can seem overly demanding in low-risk research that involves a low likelihood of experiencing physical, psychological or other harm (e.g. a single blood draw), or in research where the risks are thought to be outweighed by the potential clinical benefits for participants (e.g. a comparative effectiveness study of two established treatments with no other research procedures).

To ease the burden, some research ethicists argue that the standards for obtaining informed consent should be adapted to the risk-benefit profile of the research. Gopal Sreenivasan contends that understanding a trial's risk-benefit ratio is not required when the risks of the study are outweighed by the potential clinical benefits for participants. ${ }^{9}$ Franklin Miller and Alan Wertheimer take this idea one step further, arguing that defective understanding does not invalidate consent to studies comparing clinically indicated treatments. ${ }^{10}$ Robert Truog and colleagues question the need to seek specific consent altogether in certain randomised controlled trials of licensed interventions, ${ }^{11}$ and others are currently extending this idea to comparative effectiveness research. ${ }^{12}$ Recent proposals for regulatory reform seem to embrace these ideas. For example, the European Clinical Research Infrastructures Network suggests disclosing no more than light information' in trials with marketed drugs that are used for a new indication. ${ }^{13}$ All this suggests that consent standards need not be as rigorous when the research has a favourable risk-benefit profile for the individual participant.

However, current discussions are limited insofar as they only pertain to 'win-win' scenarios in which the research is expected to clinically benefit current participants as well as benefiting future patients. But more difficult questions arise about the permissibility of adapting consent to risk when a study offers participants no prospect of clinical benefit. Furthermore, adapting the standards for disclosure and understanding to risk might suggest that participants can be enrolled into research without their autonomous authorisation when the research is unlikely to pose serious harm to them. ${ }^{2}$ Some consequentialists may be unconcerned about this when studies are socially valuable. But a question naturally arises for deontologists: how can the plausible intuition that consent standards should be adapted to the level of risk posed by a study be reconciled with the impermissibility of enrolling participants into research without their autonomous authorisation?

Some commentators are sceptical that the two can be brought together. For example, Miller and Wertheimer have recently argued that the standard 
view of informed consent as autonomous authorisation does not have the conceptual resources to be adapted to risk. ${ }^{10}$ If correct, their argument implies that current regulations and guidelines, as well as recent proposals for regulatory reform, rest on shaky normative ground. It also suggests that any attempt to promote risk-adapted consent inherently conflicts with the standard view of informed consent.

In this paper, we argue that the standard view of consent as autonomous authorisation can be reconciled with the plausible intuition that requirements for the informed consent process should be adapted to the risks posed to participants. We assume the standard view of risk as the likelihood of experiencing harm, as well as standard frameworks for evaluating the risks of research interventions and studies; these frameworks require investigators, research ethics committees (REC) and others to evaluate the risks and potential benefits (if any) of individual research interventions before judging the aggregate level of risk posed by the study as a whole. ${ }^{14} 15$ We show that investigators can often discharge their duties in an abridged consent process when the risks of research participation are low, while a more rigorous consent process is required as the risks of the research increase. Importantly, our argument applies to studies whose risks are not, or not entirely, outweighed by the potential clinical benefits for the participant. Our argument also has important practical implications. It shows that current and proposed consent standards are not adequately calibrated to risk and, interestingly, that consent standards also need to be adapted to factors other than risk.

\section{THE PURPOSE OF INFORMED CONSENT}

Consider a simple natural history study that involves undergoing a single blood draw. When the risk of research participation is so low, why is it important to obtain valid informed consent? The answer is simple: because competent adults have autonomy rights. That is to say, they have the right to control certain aspects of their own lives, which includes the right to control what happens to their bodies. Their right to bodily control generates a duty on others not to control decisions about their bodies or trespass on them without their permission.

This explanation is at the heart of the concept of informed consent that underlies the standard view found in most research regulations and guidelines: informed consent as autonomous authorisation. ${ }^{1}$ 5-8 According to Ruth Faden and Tom Beauchamp, who have developed the most influential conception of this view, '....informed consent is rooted in concerns about protecting and enabling autonomous or self-determining choice by patients and subjects.' (ref. 2, p.235).

Autonomy rights protect competent adults from unwanted interference and they also give them the opportunity to live their lives in accordance with their own interests, preferences and values. Competent adults have the power to exercise their autonomy rights and permit, among other things, bodily contact. By giving valid consent, they can permit acts that would otherwise be rights violations. For example, if an investigator draws a participant's blood without his valid consent, she violates his right to bodily control. But no such rights violation occurs if the participant gives valid consent to this procedure.

Consent only has this morally transformative power if it is valid, and to be valid the competent person giving consent must do so voluntarily and in enough understanding of the act being consented to. This implies that a token of consent is invalid if it is proffered by a mentally incompetent individual, given in substantial ignorance or obtained as the result of coercion, deception or fraud.
This standard view of informed consent should be familiar to most readers. But what often goes unnoticed is the connection between the disclosure requirement and the voluntariness (or non-control) requirement for valid informed consent. Even Beauchamp, the chief exponent of the standard view, claims that commentators have given too much emphasis to disclosure and not enough to voluntariness. ${ }^{16}$ Disclosure is important because of its relationship to control. Illegitimately controlling a participant's enrolment decision can render the resultant token of consent involuntary and thereby invalid. The most widely discussed forms of illegitimate control are coercion, intentional deception and manipulation. But there are subtler forms of control, and these relate to inappropriate disclosure.

To see this, notice that in some contexts, the act being consented to is familiar enough to rescind the duty to inform the person giving consent. For example, if you ask your hairdresser to take a couple of inches off your ends, you convey enough understanding of what it means to get a haircut; she does not have to explain that procedure to you. The research context is different, however. Various facts about a given study might influence a potential participant's enrolment decision, but he cannot be expected to know these facts on his own. For example, the purpose of the study might make a difference to his decision, but he cannot know this until it is disclosed to him. When an investigator fails to appropriately disclose information that might influence a participant's enrolment decision, she (advertently or inadvertently) exercises control over his decision and thereby fails to respect his right not to be illegitimately controlled in the consent process. ${ }^{17}$ This illustrates that in addition to not coercing or unduly influencing potential participants, investigators have another duty not to exercise illegitimate control in the informed consent process: they must not withhold relevant information or disclose such information in an incomprehensible way. ${ }^{\mathrm{i}}$

\section{RESPONSIBILITIES IN THE INFORMED CONSENT PROCESS}

When the purpose of informed consent is properly understood, it becomes clear that investigators' responsibilities in the informed consent process are not as burdensome as is often supposed. Additionally, potential participants have responsibilities if they want to make good decisions about study participation.

\section{Investigators' responsibilities}

Regardless of the risk level of the research, investigators have a duty not to illegitimately control a potential participant's enrolment decision. This implies that they must guard against the ways in which they can undermine or reduce the voluntariness of this decision. In addition to avoiding acts of coercion, deception and certain forms of manipulation, discharging this duty involves disclosing relevant information in an understandable way and in an appropriate situational context. ${ }^{17}$ Appropriate disclosure requires investigators to disclose all those facts that they know about the research, and have reason to believe would be relevant to the participant's enrolment decision. This should include 'known unknowns', e.g. the possibility of unknown side effects of an investigational drug, or uncertainty about its riskbenefit profile. Moreover, the manner in which information is

'To improve readability, we refer to investigators' responsibilities although nurses, study coordinators and others may be responsible for obtaining consent. We also recognise that duties in the informed consent process are shaped and shared by policy makers, RECs and others responsible for ensuring the ethical conduct of research. 
disclosed is as important as the information itself. An investigator would exercise illegitimate control over a participant's enrolment decision if she disclosed information in a foreign language, or using complicated scientific terminology. Disclosure is, therefore, only appropriate if information is disclosed in a manner that gives a prospective participant a fair opportunity to understand. Finally, since investigators cannot know exactly what would influence an individual's enrolment decision, they must give potential participants the opportunity to ask questions and answer their questions honestly. ${ }^{17}$

However, discharging the duty not to illegitimately control a participant's enrolment decision does not require investigators to ensure that a potential participant understands everything that ought to be disclosed to him. Nor does it require investigators to ensure that the participant makes a decision that best protects his particular interests, preferences and values. After all, not ensuring that he has achieved this advanced level of understanding does not amount to exercising illegitimate control over his enrolment decision.

At the same time, investigators have a duty not to accept a token of consent from a person who is substantially ignorant of what they are authorising or who lacks capacity. This implies that investigators must form a reasonable belief that a potential participant has capacity and has achieved the requisite level of understanding before accepting his consent. ${ }^{18}$ There are some uncertainties about exactly what participants need to understand for consent to be valid. Yet, according to the standard view of informed consent as autonomous authorisation, '[a]t minimum, persons understand only if they have acquired pertinent information and have relevant beliefs about the nature and consequences of their actions. Their understanding need not be complete, because a grasp of the material facts is generally sufficient...' (ref. 16, p.68).

Unless there is evidence that a potential participant lacks decision-making capacity, investigators are justified in assuming his competence. That is, it is widely accepted that investigators do not need to test capacity unless there is reason for doubt. We believe that the same is-or should be-true when assessing the requirement of material understanding in the context of uncomplicated and low-risk research.

To illustrate this point, consider the informed consent process for a natural history study that involves three blood draws with no prospect of clinical benefit. The investigator has a duty to inform potential participants in clear and simple language about the purpose of the study, the three blood draws and their associated risks, the collection of anonymised personal medical information and the absence of negative implications for their clinical care if they decide against enrolment. But if a potential participant agrees to enrol in the study, the investigator can take his token of consent as valid without testing his understanding. To test his understanding of what it means to have his blood drawn after this has been explained to him would be tantamount to testing his capacity to give consent. When a potential participant shows no signs of cognitive impairment and the material information about the study is easily comprehensible, an investigator is permitted to presume that the participant has achieved adequate understanding.

\section{Potential participants' responsibilities}

By virtue of having the right to control their own lives, competent adults are free to make poor decisions. Others should respect these decisions unless they are the products of illegitimate control or substantial ignorance. ${ }^{19-21}$ By implication, if a potential participant wants to make a good enrolment decision, he also has responsibilities in the informed consent process.

As a prudential decision maker, a potential participant must ensure that he has all the necessary information to make a decision consistent with his interests, preferences and values. Investigators are responsible for tailoring the information disclosed to the informational needs of the person thinking about study enrolment. Since the investigator cannot know what facts will be dispositive for the given person, disclosure should include those facts that the study population usually finds relevant, in addition to any other facts that the investigator believes this particular person would find relevant. This information will suffice for most people, but it may be incomplete when a potential participant has unusual preferences and values.

Moreover, if a potential participant wants to best protect his own interests, then he has a responsibility to ensure that he understands the information disclosed to him. There are obvious perils of agreeing to enrol in a study without material understanding, despite being perfectly capable of comprehension. Giving lazy consent puts the participant at risk of incurring harm if the investigator non-culpably takes his consent to be validthat is, if the investigator reasonably (but mistakenly) assumes that the participant has achieved adequate understanding. Giving lazy consent also puts the participant at risk of not being able to benefit from study participation when his failure to achieve adequate understanding results in exclusion from the study.

\section{CAN INFORMED CONSENT BE ADAPTED TO RISK?}

With the responsibilities clarified, it is now possible to explain how informed consent standards can be adapted to the risks of the research. We have argued that when research is low-risk and otherwise simple, investigators' responsibilities in the informed consent process are not as burdensome as is often assumed. We now argue that, as the risks of research increase, so do concerns about the validity of consent given by the average potential participant. In turn, this argument reveals that the informed consent process should be adapted to risk and also to other factors, such as the controversy surrounding study goals or the complexity of the information disclosed, when that information is material to a potential participant's enrollment decision.

\section{Risk and the validity of consent}

The risk-adapted argument starts from the well-known empirical evidence on participants' understanding of research risk.

A wide range of studies surveying apparently competent adults enrolled in research around the world show that many fail to understand the risks of study participation. ${ }^{22-24}$ In one interview study, 63 out of 263 clinical research participants reported no risk of any sort from the research and 37 only reported the risks of standard treatment provided as part of the research..$^{25}$ Similarly, in a recent literature review, 14 out of 23 studies found that $\leq 50 \%$ of participants could recognise or name trial side effects and risks. By comparison, most studies reported adequate understanding of the study purpose, study design and the right to withdraw in the majority of participants. ${ }^{23}$ Low numeracy skills in the population-even among the highly educated-heighten concerns about otherwise competent participants' understanding of research risk. ${ }^{26}$ The same applies to widespread biases in the perception of risk. For example, familiar activities often seem less risky than they really are. ${ }^{27}$ Additionally, patient participants frequently have difficulty evaluating risks in relation to the potential clinical benefits of the research. Many are optimistic, often unreasonably so, about their potential to benefit from the research and tend to discount 
its risks. ${ }^{22}$ Many also suffer from a 'therapeutic misconception'-a mistaken belief about the nature of research, especially regarding the lack of individualised treatment, that typically results in underestimating risks and overestimating benefits. ${ }^{28}$ It can be difficult to distinguish between mistaken beliefs about the prospect of benefit and positive attitudes towards the future, ${ }^{29}$ and some of the empirical studies on consent have methodological weaknesses (e.g. potentially measuring recall of information rather than understanding ${ }^{22}$ ). Nonetheless, these data cast doubt on investigators' ability to communicate information about research risk clearly and participants' understanding of this often complex and abstract information.

On the standard view of informed consent as autonomous authorisation, material understanding is a necessary condition, ${ }^{16}$ and the risks associated with an activity are material to most people's decisions about whether to engage in that activity. Usually, as the risks of an activity increase, a rational person's willingness to take part in that activity decreases. This applies especially to activities that pose risk but offer no prospect of direct benefit. When this consideration is coupled with the fact that many research participants have a poor understanding of the risks of study participation, those willing to enrol in highrisk studies are more likely to have misunderstood the risks of the research than those willing to enrol in low-risk studies. This gives investigators reason to doubt that the consent given by candidates for high-risk research is valid.

Doubts about the validity of consent in high-risk research are exacerbated by the fact that riskier research is often more difficult to understand than research involving lower risks. For example, high-risk studies often include numerous research procedures whose risks can have complex additive and interactive effects. Moreover, riskier procedures and studies typically involve a higher likelihood of experiencing moderate or serious harm than low-risk research, and the inevitable uncertainty around likelihood estimates therefore becomes both more relevant and more confusing.

\section{Risk-adapted standards for the informed consent process}

Our analysis of informed consent implies that an investigator should not exercise illegitimate control in the informed consent discussion and only accept a token of consent when she reasonably believes that a potential participant has achieved material understanding. When the research is low-risk and otherwise simple, investigators can discharge their duties in the informed consent process by properly disclosing relevant information and obtaining a token of consent. But more is required to discharge the duty not to exercise illegitimate control over participants' enrolment decisions as the risks of research increase.

Investigators cannot merely assume that consent is valid after appropriate disclosure in moderate or high-risk research; they need to verify it and shape the informed consent process such that it promotes understanding. In order not to exercise illegitimate control over potential participants' enrolment decisions, investigators should consider the available evidence on how best to communicate risk, uncertainty and information about research more generally. ${ }^{22}{ }^{30-32}$ Although some of this evidence is mixed, there are several reliable approaches. For example, data support using plain language and pictographs, conveying risk information in frequencies rather than percentages and adopting a less is more' approach to selecting information. ${ }^{30}$ Investigators should also consider how the situational context of the consent discussion might influence uptake of risk information.

Furthermore, to prevent investigators from accepting a token of consent when a potential participant lacks material understanding, investigators should test comprehension as the risks of the research increase. Informal testing may be sufficient in research involving moderate risks, whereas formal quizzing is likely required in high-risk research. ${ }^{18}$ For example, in a study involving a research-only lumbar puncture, the investigator might ask the participant informally to explain why he is willing to enrol in the study given the risk-benefit profile.

In high-risk research, it can also be appropriate to delegate the consent discussion to an independent colleague in order to avoid misperception of study risks and benefits. For example, to obtain consent for a phase 1 trial of a novel brain cancer treatment with a research-only lumbar puncture, a qualified professional who is independent of the study might test the potential participant's understanding with a set of validated questions before obtaining his consent. Several studies have explored how to formally test understanding of material facts and participants' motivations. ${ }^{22} 33$ These additional risk-adapted duties in the informed consent process help to ensure valid consent, protect participants in high-risk research, safeguard investigators' professional integrity and promote public trust in research.

\section{FACTORS BEYOND RISK}

Exploring the ways in which risk can undermine the validity of consent allows us to identify other factors that might have the same effect. These factors are often, but not always, associated with risk. Just as investigators have additional responsibilities in the informed consent process as the risks of research increase, so too do they have additional responsibilities as studies pursue increasingly controversial goals. On occasion, the complexity of study inventions can also change investigators' responsibilities.

\section{Controversy}

The purpose of a study is often material to a participant's enrolment decision. Just as a rational person's willingness to participate in research is likely to decrease as the risks of research increase, so a person's willingness to participate in research is likely to fluctuate depending on the purpose of the research. For example, some people may not wish to contribute to research whose results may be misunderstood or abused. Examples are studies on the genetic determinants of alcoholism that could exacerbate stigma and discrimination of already marginalised groups, or studies on bioterrorist defence that could actually facilitate terrorist attacks. Given that certain studies are expected to be the subject of reasonable disagreement among the targeted research population, willingness to participate in such research is more likely to reveal a misunderstanding or confusion about its purpose than willingness to enrol in uncontroversial research. By extension, the consent of candidates for controversial studies is less likely to satisfy the requirement of material understanding, and hence, less likely to be valid on the standard view of informed consent. ${ }^{2}$ Furthermore, enrolment in such studies may constitute a significant setback to a participant's invested interests or values.

Investigators should, therefore, consider whether a study is likely to be controversial in the target population. This should include consideration of whether the research might violate religious or other deeply held beliefs, such as a study of porcinederived products in predominantly Muslim, Hindu or Sikh populations. ${ }^{35}$ When a study is expected to be controversial, investigators should informally or formally test whether participants comprehend what they are signing up for. However, when a potential participant may consider a study controversial due to his idiosyncratic preferences and interests, it is his responsibility 
to ensure that he has all the necessary information to protect these preferences (see above).

\section{Complexity}

The empirical literature finds that the average competent participant has difficulty understanding the risks of study procedures as well as complex research interventions. This pertains especially to randomisation. ${ }^{22} 23$ For example, in the recent literature review, 11 out of 17 studies reported that $\leq 50 \%$ of participants in randomised controlled trials understood their treatment would be determined by chance. ${ }^{23}$ These data give cause for concern when randomisation is material to a potential participant's enrolment decision-for example, when a participant has joined a placebo controlled study because he falsely believes that he will be selected for the experimental treatment. In such cases, investigators cannot assume that consent is valid after appropriate disclosure. In order not to exercise illegitimate control over potential participants' enrolment decisions, investigators may have to use or develop methods for communicating complex interventions that are commonly misunderstood. For example, participants judge some descriptions of randomisation to be clearer than others. ${ }^{34}$ Furthermore, investigators may need to test understanding to ensure that they have disclosed complex information in an understandable way.

Although controversy and complexity can influence investigators' responsibilities in the informed consent process, risk is typically the most important factor. Most research studies pose some level of risk to participants, while only a few studies pursue potentially controversial goals. Thus, context-dependent consent standards will generally give greater emphasis to risk than to other factors. Moreover, complexity is not of concern per se, but only when we have reason to suspect that, had the potential participant understood the complex intervention, he would not have enrolled in the study.

\section{THEORETICAL AND PRACTICAL IMPLICATIONS}

Drawing on the standard view of informed consent as autonomous authorisation, ${ }^{216}$ we have shown that concerns about the validity of consent increase as the research becomes increasingly risky, controversial and (in some cases) complex. Our arguments support adopting an approach to consent that adapts investigators' responsibilities in the consent process to risk and these other factors. This analysis has important theoretical and practical implications.

In theoretical terms, our analysis shows that the standard view of informed consent can be adapted to risk, and that it can do justice to the transactional nature of consent. Miller and Wertheimer have recently argued that the autonomous authorisation model of consent needs to be replaced because it cannot accommodate risk-adapted criteria for the validity of consent. ${ }^{10}$ Given that the standard view of consent is enshrined in most research regulations and guidelines, ${ }^{15-8}$ their argument would require a sweeping overhaul of current practice. Fortunately, our analysis suggests that we do not have to go there.

However, this is not Miller and Wertheimer's only concern. They also worry that informed consent as autonomous authorisation jeopardises socially valuable research in failing to give investigators fair notice about when consent can be considered valid. ${ }^{10}$ If autonomous authorisation cannot make sense of transactional fairness, it threatens the other values that are served by consent, such as making mutually beneficial arrangements that would otherwise be impermissible. We show that this concern is unfounded. We derive conditions for a fair consent transaction (see the section on Risk-adpated standards for the informed consent process) and specify the consenting parties' respective responsibilities in the informed consent process (see the section on Responsibilities in the informed consent process).

In practical terms, our analysis reveals that consent standards should be adapted to risk and also to controversy, occasionally complexity, and possibly other factors. This is a novel insight, which will help to develop a nuanced and robust approach to how investigators should discharge their responsibilities in the informed consent process. Further work is necessary to systematically evaluate which factors risk undermining the validity of consent on our analysis. However, the available evidence suggests that participants currently have most difficulty processing risk and complex or abstract information. ${ }^{23}$ Importantly, because our argument for risk-adapted consent standards starts from the empirical data on participant comprehension, investigators' responsibilities in the informed consent process will be dynamic. As investigators learn to better communicate risk, their responsibilities will become less demanding. Conversely, novel research methods may introduce complex interventions or concepts that could make investigators' responsibilities more exacting.

Furthermore, our analysis suggests that the current discussion about risk-adapted informed consent is incomplete. Research ethicists $^{9} \quad 10 \quad 11$ and stakeholders in research ${ }^{13}$ focus almost exclusively on relaxing consent standards in low-risk research. However, as our argument shows, a comprehensive risk-adapted perspective on informed consent requires shedding consent requirements in low-risk and otherwise simple research, while adding safeguards for consent to higher-risk and more controversial research.

\section{OBJECTIONS AND REPLIES Grades of consent}

Adapting the standard view of consent to risk might be seen as diluting the concept of consent. It appears as if investigators only have to obtain low-grade consent in low-risk research, while something more substantial is required as the risks of the research increase. Why call what is acquired after mere appropriate disclosure 'valid consent' when what is obtained after testing understanding and voluntariness seems so much more morally robust?

This objection misses the deontological constraints present in the standard view of consent that have guided our argument. Regardless of a study's risk level, investigators have a duty not to exercise forms of illegitimate control over a potential participant's enrolment decision, as well as a duty to test understanding of material information when there is reason to suspect a lack of understanding. As we have argued, discharging these duties is often much harder in research involving higher risks. The additional responsibilities help to ensure that investigators obtain valid informed consent when higher risk, controversy and perhaps other aspects of the research such as complexity may compromise the validity of consent. This implies that investigators' additional responsibilities apply even when the research is low-risk, but otherwise controversial or (in some cases) complex.

\section{Unnecessary}

Not all commentators will agree with recent critics that informed consent as autonomous authorisation is insensitive to risk; some may, therefore, consider our analysis unnecessary. The autonomous authorisation view of consent assumes a reasonable person standard of disclosure. ${ }^{2}$ Since a reasonable person is likely to judge low risks to be less material to her 
enrolment decision than high risks, the autonomous authorisation view could recommend adapting the informed consent process accordingly. This would imply an abridged disclosure and, consequently, a lower threshold for understanding in low-risk research, as well as a more thorough disclosure and higher requirement of understanding in high-risk studies.

We agree that informed consent as autonomous authorisation can be adapted to risk in this way, but our analysis shows that deriving a risk-adapted informed consent process solely from the reasonable person standard of disclosure is likely to compromise the validity of consent. By determining what ought to be disclosed based on what a reasonable person would want to know, investigators may withhold potentially relevant information from the actual person giving consent. Actual participants perceive risk in different ways, and some may view the risks associated with a study differently than a reasonable person. For actual participants to be able to judge whether study risks are appropriate for them, they must be given comprehensive information about these risks. Our analysis explains how and why investigators ought to disclose a good deal of information to potential participants, but need not test understanding of that information when studies involve low risks. It, therefore, guards against ways of adapting consent as autonomous authorisation to risk that are likely to compromise the validity of consent.

\section{Too burdensome}

Our argument could be seen as increasing the burden on investigators by adding to their duties in the informed consent process. After all, we are proposing that investigators sometimes delegate consent discussions and formally test participants' understanding, neither of which is currently required (although sometimes practiced, e.g. ref. 36).

Balancing appropriate subject protection with the need to minimise the burden of conducting socially valuable research requires being attentive to those factors that might compromise the validity of consent. Our analysis indeed reveals that investigators have more demanding responsibilities in research involving higher risk. However, minimal risk research represents between $40 \%$ and $50 \%$ of new protocols for ethical review. ${ }^{37} 38$ When minimal risk studies are otherwise simple and uncontroversial, our analysis implies that it is permissible to obtain a token of consent after mere appropriate disclosure. If a more robust consent process is required to ensure the ethical conduct of high-risk studies, we are prepared to defend the additional burden, even if it would delay or stifle some valuable research. Current guidelines and regulations largely fail to specify the factors that can undermine the validity of participants' consent. Our analysis alerts investigators to these factors and specifies responsibilities to mitigate their influence on participants' enrolment decisions, thereby helping to close a gap in current subject protections.

With this said, future empirical research will likely show that investigators' overall responsibilities are considerably lighter on our analysis than on the standard view of informed consent. Determining what ought to be disclosed should itself be informed by careful analysis of empirical research on what potential participants generally want to know. Studies show that participants in minimal risk research seek less information than is currently listed on informed consent documents. ${ }^{39}{ }^{40}$ Furthermore, the available data suggest that shorter and simpler consent forms facilitate understanding or do not compromise it. ${ }^{41-43}$ Currently, many consent forms are excessively long, replete with legal boilerplate and written at too high a reading level for the intended reader. ${ }^{44}$ Giving participants a fair opportunity to understand the information that ought to be disclosed to them likely involves shortening and simplifying consent forms.

\section{Nothing new}

Critics might regard our abridged informed consent process in low-risk research as being already implemented. Anecdotal evidence suggests that it is common practice for investigators to have brief informed consent discussions when a study poses only limited risks. Furthermore, some research regulations allow modifications of some or all elements of informed consent in minimal risk research. ${ }^{8}$

However, this practice is not consistent with the standard view of consent as autonomous authorisation set out in most research regulations and guidelines. ${ }^{1}{ }^{5-8}$ The existing literature suggests that some investigators indeed discuss less information than is normally required. ${ }^{46}$ But the worry is that investigators inadvertently exercise illegitimate control over participants' enrolment decisions by omitting information they ought to disclose. Moreover, when investigators fail to explain poorly understood concepts in a clear and understandable way, they fail to disclose appropriately and they may even inadvertently violate the material understanding requirement for valid informed consent. For example, only 65 of 117 investigators in a multinational randomised controlled trial emphasised issues related to randomisation, ${ }^{47}$ a concept that is sometimes material to enrolment decisions and often poorly understood. ${ }^{22-24}$ Our analysis explains and justifies why an abridged informed consent process in low-risk research is acceptable and offers a framework to systematically specify investigators' responsibilities in the informed consent process. This gives a proper normative grounding for those aspects of current practice that are justifiable, but helps to correct problematic practices.

\section{Incomplete}

Critics might agree that current fascination with risk-adapted standards for informed consent can distract from other factors that potentially compromise the validity of consent. But they might object that our list of additional factors is not exhaustive.

Our analysis is not designed to be exhaustive. It is just designed to show that the view of informed consent found in most of the guidelines and regulations can be adapted to risk, and the arguments that support risk adaptation also support sensitivity to other features of research.

Furthermore, our analysis underscores the normative significance of empirical work conducted on informed consent. All too often, the value of this research is seen primarily in its use in challenging the standard view of consent. But this researchand research in related fields, such as health communication and cognitive psychology-is an invaluable source of information for identifying other features of medical research with the potential to undermine the validity of consent. Such research helps to specify how investigators can better discharge their obligations in the consent process, and consequently allows potential participants to make better enrolment decisions.

\section{Too complicated}

To implement risk-adapted consent standards, it is necessary to classify risks to participants in categories (e.g. low, moderate, high) and specify risk-adapted consent standards for each category. Practically minded critics will likely object that such classifications are notoriously complex and controversial, ${ }^{13} 14$ and developing and implementing risk-adapted consent standards is therefore difficult. Moreover, risk-adapted standards can falsely 
suggest that risk is the main or only factor that influences the informed consent process.

These are genuine concerns and need to be addressed. Risk categories should be defined as precisely as possible and illustrated with evidence-based examples, and there should be clear guidance on how to apply the categories. Ideally, a collection of examples would cover the most common research interventions in each category. These examples could then be used as default or paradigm judgements about risk, to be interpreted by investigators and RECs in relation to the protocol under consideration. Moreover, new risk categories should be tested before they are adopted and stakeholders educated. Finally, it is critical to set out clearly how other factors (e.g. controversy) influence informed consent standards.

All this is a tall order, and residual uncertainties about how to classify risks will likely remain. But the benefits of risk-adapted consent standards arguably outweigh the disadvantages; not adapting the informed consent process to risk and other factors results in a 'one-size-fits-all' approach to consent, which can unnecessarily delay or stifle valuable research.

\section{CONCLUSION}

A proper understanding of the standard view of informed consent reveals that consent is more likely to be invalid as the risks of research increase. This implies that the informed consent process should be adapted to risk. However, risk is not the only factor that can compromise the validity of consent to medical research participation. Other factors, such as the potentially controversial nature of the research or the complexity of the information disclosed, also may invalidate consent. This paper explains and justifies why investigators' responsibilities in the informed consent process should be adapted to risk, complexity, controversy and potentially other factors. Our argument abides by the deontological constraint that study participants must give valid informed consent to invasive research interventions, whether or not the risks of these interventions are significant. At the same time, it captures the plausible intuition that consent standards should be less rigorous in low-risk research. The paper, therefore, offers the lacking normative justification for the risk-adapted consent standards, which significantly ease the burden on investigators in low-risk research while offering missing guidance on how to obtain valid consent to research involving higher risks.

Acknowledgements We thank Bob Goodin, Barbara Koenig, Frank Miller, Joseph Millum, David Wendler, and Alan Wertheimer for comments on previous versions of this paper. We are also grateful for the suggestions from audiences at the $\mathrm{NIH}$ Department of Bioethics, the WHO Ethics and Health Unit, and the 2012 Society of Applied Philosophy meeting.

Contributors The authors jointly developed the argument, wrote, reviewed and revised the manuscript, and approved the final manuscript as submitted. Both authors contributed equally to the paper.

Funding Our work on this paper was supported by the Brocher Foundation. Annette Rid received funding from the People Programme (Marie Curie Actions) of the European Union's Seventh Framework Programme (FP7/2007-2013) under REA grant agreement $n^{\circ} 301816$.

Competing interests None.

Provenance and peer review Not commissioned; externally peer reviewed.

\section{REFERENCES}

1 Emanuel EJ, Wendler D, Grady C. What makes clinical research ethical? JAMA 2000;283(20):2701-11

2 Faden RR, Beauchamp TL, King NM. A history and theory of informed consent. New York, NY: Oxford University Press, 1986.

3 Levine RJ. Ethics and regulation of clinical research. New Haven, CT: Yale University Press, 1988.
4 Berg JW, Appelbaum PS, Lidz CW, et al. Informed consent: legal theory and clinical practice. Fair Lawn, NJ: Oxford University Press, 2001.

5 Council for International Organizations of Medical Sciences (CIOMS) in collaboration with the World Health Organization (WHO). International Ethical Guidelines for Biomedical Research Involving Human Subjects. Switzerland: Geneva, 2002.

6 World Medical Association, (WMA). WMA Declaration of Helsinki - Ethical Principles for Medical Research Involving Human Subjects. Brazil: Fortaleza, Oct 2013.

7 European Parliament, Council of the European Union. Directive 2001/20/EC of the European Parliament and of the Council of 4 April 2001 on the approximation of the laws, regulations and administrative provisions of the Member States relating to the implementation of good clinical practice in the conduct of clinical trials on medicinal products for human us. Official J Eur Commun 2001; L121:34-44.

8 U.S. Department of Health and Human Services. U.S. Code of Federal Regulations, 45 CFR 46; revised 1991, 1991.

9 Sreenivasan G. Does informed consent to research require comprehension? Lancet 2003;362:2016-18.

10 Miller FG, Wertheimer $A$. The fair transaction model of informed consent: an alternative to autonomous authorization. Kennedy Inst Ethics J 2011;21:201-18.

11 Truog RD, Robinson W, Randolph A, et al. Is informed consent always necessary for randomized, controlled trials? New Engl J Med 1999;340:804-7.

12 Faden RR, Beauchamp TL, Kass NE. Informed consent, comparative effectiveness, and learning health care. N Engl J Med 2014;370:766-68.

13 European Clinical Research Infrastructures Network (ECRIN), on behalf of the "Roadmap Initiative for Clinical Research in Europe". Multidisciplinary Workshop on Risk Based Approach in Clinical Trials (18th January 2010, Barcelona, Spain).

14 Rid A, Wendler D. Risk-benefit assessment in medical research-critical review and open questions. Law Probability Risk 2010;9(3-4):151-77.

15 Rid A, Wendler D. A framework for risk-benefit evaluations in biomedical research. Kennedy Inst of Ethics J 2011;21(2):141-79.

16 Beauchamp TL. Autonomy and consent. In: Miller FG, Wertheimer A, eds. The ethics of consent: theory and practice. New York, NY: Oxford University Press, 2010:55-78.

17 Bromwich D, Millum J. Disclosure and consent to medical research participation. J Moral Philos 2015;12:195-219.

18 Wendler D. How to enroll participants in research ethically. JAMA 2011;305: 1587-8.

19 Kleinig J. The nature of consent. In: Miller FG, Wertheimer A, eds. The ethics of consent: theory and practice. New York, NY: Oxford University Press, 2010:3-24.

20 Dworkin R. Life's dominion : an argument about abortion, euthanasia, and individual freedom. 1st edn. New York, NY: Knopf, 1993.

21 Feinberg J. Harm to self. New York, NY: Oxford University Press, 1986.

22 Flory J, Wender D, Emanuel EJ. Empirical issues in informed consent for research. In: Emanuel EJ, Grady C, Crouch RA, et al., eds. The Oxford Textbook of Clinical Research Ethics. New York, NY: Oxford Univeristy Press, 2008:645-60.

23 Mandava A, Pace C, Campbell B, et al. The quality of informed consent: mapping the landscape. A review of empirical data from developing and developed countries. J Med Ethics 2012:38:356-65.

24 Cox AC, Fallowfield LJ, Jenkins VA. Communication and informed consent in phase 1 trials: a review of the literature. Support Care Cancer 2006;14:303-9.

25 Lidz CW, Appelbaum PS, Grisso T, et al. Therapeutic misconception and the appreciation of risks in clinical trials. Soc Sci Med 2004;58:1689-97.

26 Gigerenzer G, Gaissmaier W, Kurz-Milcke E, et al. Helping doctors and patients make sense of health statistics. Psychol Sci Public Interest 2008;8:53-96.

27 Slovic P. The perception of risk. London, Sterling VA: Earthscan, 2000.

28 Appelbaum PS, Lidz CW. The therapeutic misconception. In: Emanuel E, Grady C, Crouch RA, et al., eds. The Oxford Handbook of Clinical Research Ethics. New York, NY: Oxford University Press, 2008:633-44.

29 Horng S, Grady C. Misunderstanding in clinical research: distinguishing therapeutic misconception, therapeutic misestimation, and therapeutic optimism. IRB 2003;25:11-16

30 Fagerlin A, Zikmund-Fisher BJ, Ubel PA. Helping patients decide: ten steps to better risk communication. J Nat/ Cancer Inst 2011;103:1436-43.

31 Politi MC, Han PK, Col NF. Communicating the uncertainty of harms and benefits of medical interventions. Med Decis Making 2007;27:681-95.

32 Palmer BW, Lanouette NM, Jeste DV. Effectiveness of multimedia aids to enhance comprehension of research consent information: a systematic review. IRB 2012:34:1-15.

33 Appelbaum PS, Lidz CW, Klitzman R. Voluntariness of consent to research: a preliminary empirical investigation. IRB 2009;31:10-14.

34 Jenkins V, Fallowfield L, Cox A. The preferences of 600 patients for different descriptions of randomisation. Brit J Cancer 2005;92:807-10.

35 Eriksson A, Burcharth J, Rosenberg J. Animal derived products may conflict with religious patients' beliefs. BMC Med Ethics 2013;14:48.

36 Sugarman J, Corneli A, Donnell D, et al. Are there adverse consequences of quizzing during informed consent for HIV research? J Med Ethics 2011; 37:693-7.

37 Kim S, Ubel P, De Vries R. Pruning the regulatory tree. Nature 2009;457:534-5. 


\section{Research ethics}

38 Sugarman J, Getz K, Speckman JL, et al. The cost of institutional review boards in academic medical centers. New Engl J Med 2005;352:1825-7.

39 Desch K, Li J, Kim S, et al. Analysis of informed consent document utilization in a minimal-risk genetic study. Ann Intern Med 2011;155:316-22.

40 Antoniou EE, Draper $\mathrm{H}$, Reed K, et al. An empirical study on the preferred size of the participant information sheet in research. J Med Ethics 2011;37:

557-62.

41 Dresden GM, Levitt MA. Modifying a standard industry clinical trial consent form improves patient information retention as part of the informed consent process. Acad Emerg Med 2001;8:246-52.

42 Beardsley $E$, Jefford $M$, Mileshkin L. Longer consent forms for clinical trials compromise patient understanding: so why are they lengthening? I Clin Oncol 2007;25:13-14.
43 Rogers CG, Tyson JE, Kennedy KA, et al. Conventional consent with opting in versus simplified consent with opting out: an exploratory trial for studies that do not increase patient risk. J Pediatr 1998;132:606-11.

44 Albala I, Doyle M, Appelbaum PS. The evolution of consent forms for research: a quarter century of changes. IRB 2010;32:7-11.

45 Paasche-Orlow MK, Taylor HA, Brancati FL. Readability standards for informed consent forms as compared with actual readability. New Engl I Med 2003;348: $721-6$.

46 Williams CJ, Zwitter M. Informed consent in European multicentre randomised clinical trials—are patients really informed? Eur J Cancer 1994; 30A:907-10.

47 Sabik L, Pace CA, Forster-Gertner HP, et al. Informed consent: practices and views of investigators in a multinational clinical trial. IRB 2005;27:13-18. 